Résumés des conférences et travaux

\title{
Symbolique médiévale et moderne
}

\section{Michel Pastoureau}

\section{OpenEdition \\ Journals}

Édition électronique

URL : https://journals.openedition.org/ashp/1321

DOI : 10.4000/ashp.1321

ISSN : 1969-6310

Éditeur

Publications de l'École Pratique des Hautes Études

\section{Édition imprimée}

Date de publication : 1 octobre 2012

Pagination : 198-206

ISSN : 0766-0677

Référence électronique

Michel Pastoureau, "Symbolique médiévale et moderne », Annuaire de l'École pratique des hautes études (EPHE), Section des sciences historiques et philologiques [En ligne], 143 | 2012, mis en ligne le 25 septembre 2012, consulté le 03 août 2021. URL : http://journals.openedition.org/ashp/1321 ; DOI : https://doi.org/10.4000/ashp.1321 


\title{
SYMBOLIQUE MÉDIÉVALE ET MODERNE
}

\author{
Directeur d'études : M. Michel Pastoureau, \\ correspondant de l'Institut
}

Programme de l'année 2010-2011: Histoire naturelle et culturelle du porc dans les sociétés européennes (suite).

Le thème des conférences était prévu pour se dérouler sur deux ans. Il se proposait d'étudier la place du porc dans la culture matérielle, les savoirs zoologiques, l'imaginaire, les croyances et les sensibilités, de la protohistoire jusqu'au XVI ${ }^{\text {e }}$ siècle. Dans cette évolution de longue durée, qui conduit nécessairement l'historien sur des terrains documentaires variés, un certain nombre de grands dossiers ont spécialement retenu l'attention : le lexique et les faits de langue, l'élevage et l'alimentation, les classifications animales, la place du porc dans les croyances et les pratiques religieuses, les rejets et tabous qui l'entourent, le monde des emblèmes et des symboles.

Les conférences de l'année universitaire 2009-2010 avaient essentiellement porté sur l'Antiquité et sur le haut Moyen Âge. Celles de la présente année ont eu pour objet l'histoire sociale et symbolique du porc de l'époque féodale au $\mathrm{XVI}^{\mathrm{e}}$ siècle.

\section{Un pilier de l'alimentation médiévale}

La primauté du porc dans la partie carnée de l'alimentation médiévale a parfois été contestée. Certains archéozoologues se sont étonnés de trouver sur les sites habités qu'ils fouillaient, beaucoup plus d'os d'ovins et de bovins que de porcins. D'où des controverses, parfois âpres, pour déterminer quelle était de ces trois viandes la plus consommée. Sur les sites fouillées, la relative rareté des os de porc peut avoir pour cause l'utilisation qui en est faite pour fabriquer différents produits ou objets, notamment des colles ou de menus ustensiles. Dans l'Europe médiévale tout dans le cochon est utilisé : depuis le sang et les os jusqu'aux oreilles et aux soies, en passant par la vessie et les intestins. Ce qui n'est le cas ni du bœuf, ni du mouton. Même si ce dernier constitue lui aussi une des richesses de l'Occident médiéval.

En fait, partout, la part la plus importante de l'alimentation carnée est fournie par le cochon. Le bœuf sert surtout aux labours et à la fumure des champs : abattu âgé, sa viande est dure et peu appréciée. À l'époque féodale, et encore à la fin du Moyen Âge, rares sont les paysans qui élèvent des bovins pour la nourriture. En revanche, ils possèdent parfois quelques moutons et souvent un ou deux porcs. Presque toutes les maisons possèdent un lardier où la viande est salée ou fumée afin de pouvoir être consommée toute l'année. Cette consommation varie selon les saisons et selon ce qu'a pu procurer par ailleurs la pêche ou le braconnage, voire la volaille. Elle atteint son niveau record en octobre, novembre et décembre et son niveau le plus bas pendant le Carême. Quand le porc est tué à l'entrée de l'hiver, il est possible de manger pendant 
quelque temps de la viande fraîche (carnis recens) et non pas de la viande salée à laquelle, par nécessité, on est habitué. Cette viande fraîche est rôtie ou bouillie, rarement frite (avec du saindoux) et jamais grillée.

Grâce au lard et aux salaisons, paysans et citadins peuvent donc manger de la viande à peu près toute l'année. Le plat carné le plus ordinaire consiste à associer des pois à du lard et à manger le tout sous forme de soupe ou de bouillie. Les « jambons », c'est-à-dire les salaisons, sont une vraie richesse, soigneusement gardée et protégée. Le vol de jambons est du reste un thème littéraire fréquent, comme par exemple dans les différentes branches du Roman de Renart, véritable « roman de la faim ». De même, dans les fabliaux et dans les contes, le boucher est souvent présenté comme un affameur, un personnage cruel et néfaste qui, comme le meunier - autre personnage détesté du paysan $-\mathrm{s}$ 'enrichit injustement.

\section{Troupeaux et porchers}

Le porc est un animal facile à nourrir, bien plus que le bœuf ou même que le mouton. Vorace et omnivore, il est peu difficile sur la qualité de son alimentation, celle-ci pouvant même aller jusqu'à l'absorption de charognes, voire de fanges ou d'excréments mélangés à du son. D'où sa réputation de saleté et les tabous qui l'accompagnent. Au Moyen Âge, plusieurs auteurs soulignent le grand contraste existant entre les nourritures viles ou abjectes qu'absorbe le cochon et la saveur incomparable de sa viande; quelques-uns y voient un miracle ou un don de Dieu.

D'une manière générale, l'élevage médiéval est moins bien connu que l'agriculture proprement dite. Les documents en parlent peu avant le XIII ${ }^{\mathrm{e}}$ siècle, bien que depuis l'époque carolingienne il représente une activité essentielle dans la vie des campagnes. Il faut souvent tenter de reconstituer telle ou telle pratique d'élevage par le biais de témoignages indirects, notamment par ceux qui mettent en scène les conflits opposant pasteurs et cultivateurs : sur un terroir donné, faut-il ou non étendre la part des terres cultivées, pour nourrir une population sans cesse plus nombreuses? Cela se fait toujours au détriment de la forêt et des pâtures, donc de l'élevage. Pendant trois siècles, du début du XI ${ }^{\mathrm{e}}$ à la fin du XIII ${ }^{\mathrm{e}}$, les cultivateurs ont en général eu gain de cause. Par là même, le bétail se faisant moins nombreux - faute de terres suffisantes - il devint plus cher, et l'élevage se transforma progressivement en une affaire d'argent. Au début du $\mathrm{XIV}^{\mathrm{e}}$ siècle, élever des porcs constitue parfois déjà une véritable activité professionnelle et non plus seulement une nécessité de la vie rurale, qu'elle soit villageoise ou monastique.

À la fin du Moyen Âge, cependant, le paysage agraire se modifie de nouveau. La guerre, les épidémies et la crise démographique entraînent l'arrêt des défrichements. La forêt et la lande regagnent le terrain perdu sur les terres emblavées, et les troupeaux, ceux de moutons comme ceux de porcs, bénéficient de davantage d'espace. Malgré des difficultés de tous ordres, l'Europe peut continuer à manger de la viande. C'est à cette époque que se situent les premières tentatives pour améliorer les races : obtenir des moutons à longue laine et des porcs à chair abondante. Les moines cisterciens se montrent en ce domaine les précurseurs des physiocrates du XVIII ${ }^{\mathrm{e}}$ siècle, même si les résultats obtenus restent modestes. 
Les cochons sont parfois parqués autour de la ferme, afin qu'ils n'aillent pas vagabonder tout à loisir dans les champs ou dans les vignes. Mais, plus souvent, tous les porcs d'un village sont réunis en troupeau et placés sous la houlette d'un porcher commun qui les mène au paisson ou à la glandée. Ce porcher, sorte de «fonctionnaire » villageois, est souvent un simple d'esprit ou bien un jeune garçon particulièrement pauvre : la fréquentation quotidienne des porcs passait au Moyen Âge pour une activité dévalorisante, contrairement à celles des moutons. Lorsque les porcs sont conduits dans la forêt, le porcher ne rentre pas au village tous les soirs mais dort près de ses bêtes, dans une cabane semblable à celle du charbonnier ou du bûcheron. Ces trois personnages - le porcher, le charbonnier et le bûcheron - qui vivent solitaires au fond des bois, suscitent le mépris, la crainte ou la suspicion des paysans. Toute une littérature orale leur est consacrée qui les transforment en véritables hommes sauvages, coupables de commerce avec le diable et avec ses créatures, l'ours et le loup.

Les porchers jouent cependant un rôle important dans la vie des campagnes. On compte en général un gardien pour une soixantaine de porcs, c'est-à-dire un porcher pour une trentaine de familles. S'il est difficile d'évaluer le nombre des porcs comparé à celui des autres animaux sur un terroir donné, pour l'Angleterre des calculs précis ont pu être faits concernant la fin du $\mathrm{XI}^{\mathrm{e}}$ siècle, grâce à ce document exceptionnel que constitue le Domesday Book (sorte de cadastre général qui permettait au roi de fixer avec précision les taxes et les contingents militaires dus par chacun de ses vassaux). Chaque comté élève alors en moyenne trois fois plus de porcs que de bovins, mais aussi trois fois plus de moutons que de porcs. En 1086, le petit comté de Rutland, par exemple, situé au centre de l'Angleterre, possède exactement 1026 chevaux, 3167 bœufs, 11414 porcs et 31650 moutons, pour une population d'environ 30000 habitants. À la même époque, sur le continent, la proportion de moutons est moins grande (sauf sur le pourtour méditerranéen). Au début du XIII ${ }^{\mathrm{e}}$ siècle, la Normandie et la Bretagne, par exemple, élèvent autant de porcs que de moutons.

\section{Le porc dans la forêt}

Le porc médiéval est un animal de la forêt. C'est là qu'il trouve l'essentiel de sa nourriture, notamment les faines sous les hêtres et les glands sous les chênes. Et cela à un moment où la végétation se ralentit et où, pour les autres animaux, commencent à se poser le problème de la nourriture. L'histoire du porc au Moyen Âge est donc intimement liée à celle de la forêt. Depuis l'époque de Charlemagne, l'habitude est prise, partout en Europe, d'évaluer la taille d'un bois ou d'une forêt selon le nombre de porcs qui peuvent y être nourris en une année. À la fin du $\mathrm{x}^{\mathrm{e}}$ siècle, par exemple, il faut en région parisienne environ 150 hectares pour élever 100 porcs. Sous le règne de saint Louis, presque trois siècles plus tard, un hectare par porc (voire parfois moins d'un hectare) est devenu suffisant; mais le droit de pacage, son calendrier et le nombre des bêtes admises en forêt sont désormais soigneusement réglementés. Il en sera ainsi jusqu'à la fin de l'Ancien Régime.

Le porc a en effet sur le sol de la forêt une action plus ou moins dévastatrice. Quand il ne trouve pas suffisamment de nourriture à la surface du sol, il le fouille et, ce faisant, dénude ou détruit les racines, déterre les graines enfouies et compromet la 
repousse des herbes et des arbres. Sans être aussi nocif pour la végétation que la chèvre ou le mouton, le porc est lui aussi un animal destructeur. Tant que les forêts eurent une étendue incommensurable, à l'époque gauloise et pendant tout le haut Moyen Âge, les porcs purent y vivre librement, à demi-sauvages. Mais lorsque cette étendue se fit moins vaste et qu'à partir du XII ${ }^{\mathrm{e}}$ siècle, les bois se firent moins étendus, les porcs durent être surveillés et leur pacage fit l'objet de droits soigneusement contrôlés.

En forêt, le porc se nourrit de racines, d'herbes, de ramilles, de truffes ou de champignons, parfois de petits animaux (oisillons, limaces, rongeurs ou reptiles). Mais il mange aussi des fruits sauvages : pommes, poires, baies, noix, châtaignes et surtout faines et glands. Au pré ou sur les chemins, pendant la période où il n'est pas autorisé à se nourrir dans la forêt, il aime l'herbe tendre et le trèfle, mais il faut compléter sa nourriture avec du son, de l'orge, des sous-produits du lait ou des déchets divers. Son goût pour les rebuts et les ordures rend du reste possible son élevage en ville. En règle générale, les animaux ne peuvent manger que les fruits tombés au sol; il est interdit aux paysans de battre les branches du chêne pour en faire tomber les glands (sauf si le sol est recouvert de neige). De même, le ramassage de ces glands est rigoureusement réglementée.

Les forêts qui conviennent le mieux au pâturage des porcs, sont des forêts de chênes suffisamment vieux pour donner des glands en abondance, c'est-à-dire des chênes de plus de quarante ans d'âge. Mais ceux-ci ne donnent des récoltes pleines que tous les deux ou trois ans. Il faut donc déplacer les porcs dans la forêt au fil des années, et même au fur et à mesure qu'ils ont consommé les fruits tombés par terre afin qu'ils ne s'attaquent pas au sous-sol. D'où, partout, des zones forestières réservées au pasnage des cochons. D'où aussi des restrictions calendaires à partir du $\mathrm{XIII}^{\mathrm{e}}$ siècle, les porcs n'étant plus admis dans les forêts en toutes saisons mais seulement à l'automne. Au fil des siècles et des décennies, ces restrictions deviennent plus nombreuses, à la grande colère des villageois, dont le troupeau doit en outre passer après celui du seigneur.

Très proche de ses bêtes, le porcher a le souci de leurs intérêts. L'engraissement dans la forêt constitue parfois une véritable transhumance, qui oblige les gardiens de pourceaux à rester éloignés de leur village pendant de longues semaines. L'homme et l'animal vivent alors en totale symbiose - d'où la réputation de rusticité qu'avaient déjà les porchers dans l'Antiquité et qu'ils conservent tout au long du Moyen Âge. Souvent, le porcher n'hésite pas à conduire son troupeau sur des terres où la vaine pâture villageoise est interdite, ou bien à le faire passer avant le troupeau du seigneur, ou encore à ne pas respecter le calendrier des droits de pacage. D'où partout, au Moyen Âge, des conflits violents opposant des paysans à leur seigneur, à l'abbaye voisine, ou même, plus simplement, aux paysans du village d'à-côté. Le porc, seule vraie richesse des campagnes occidentales, est un enjeu économique, cause de jalousies et de tensions sociales.

\section{Le porc en ville}

En ville, le porc joue un rôle d'éboueur. Il se nourrit de déchets et d'ordures, ceux des maisons, des boutiques, des foires et des marchés, mais aussi ceux des cimetières. 
Au début du XIII ${ }^{\mathrm{e}}$ siècle, le roi de France Philippe Auguste dût faire entourer le cimetière des Innocents à Paris d'un mur suffisamment haut pour empêcher les porcs d'aller y déterrer les cadavres. Des décisions semblables sont connues pour York en 1243, Rouen en 1302, Bruges en 1337, Nancy en 1385, Nuremberg en 1416. En ville, le porc n'est pas vraiment élevé en porcherie; il l'est surtout dans la rue, sur les places et les terrains vagues, au bord des fleuves, voire dans les parcs ou les jardins. Depuis l'Antiquité, le porc urbain est un animal vagabond qui prend sa nourriture où il la trouve. D'où des abus, des querelles et des accidents. Nombreux sont les procès qui mettent en scène des porcs ayant visité une boutique, dévasté un jardin, pillé une réserve ou un charroi, blessé (voire dévoré) des enfants.

La divagation des porcs occasionne en effet de nombreux accidents. Le plus tristement célèbre est celui dont fut victime, en 1131, le prince Philippe, fils aîné du roi de France Louis VI le Gros, renversé de cheval par un cochon vagabond et mort des suites de cette chute. À partir de la fin du XII ${ }^{\mathrm{e}}$ siècle, toutes les villes d'Europe prennent des décisions réglementaires pour limiter ou pour interdire la circulation des porcs dans les rues. Mais le renouvellement perpétuel de ces textes prouve qu'ils n'étaient guère respectés, malgré les amendes ou les châtiments parfois très sévères qu'ils instauraient. À Paris, seuls les religieux de l'ordre de saint Antoine, les Antonistes, voués à l'action charitable et au soin des malades atteints du mal des Ardents (sorte d'épilepsie), conservèrent le privilège de laisser leurs porcs courir en liberté dans les rues. Il va sans dire que ces porcs Antonistes n'eurent jamais le monopole de la rue parisienne, et que bien d'autres cochons de toutes provenances s'y rencontrèrent jusqu'au $\mathrm{XVI}^{\mathrm{e}}$ siècle. L'édit de 1539, plus sévère encore que les précédents, semble finalement avoir eu raison de leur vagabondage. Mais à Milan et à Cologne, on trouve encore des cochons girovagues au XVIII ${ }^{\mathrm{e}}$ siècle, et à Naples, jusqu'au début du $\mathrm{XX}^{\mathrm{e}}$.

\section{Le christianisme et le porc}

L'attitude première du Christianisme à l'égard du porc est issue des traditions bibliques. Pour le Moyen Âge chrétien la Bible est non seulement le livre sacré par excellence mais aussi un ouvrage de référence scientifique, une authentique encyclopédie, spécialement utile à consulter dans le domaine de l'histoire naturelle. Elle sert de fondement à la théologie, à la prédication, à l'enseignement. Autorité suprême, elle se lit et se glose à plusieurs niveaux. Toutefois, quel que soit le point de vue envisagé, la place du porc dans les Écritures est toujours dévalorisée. Pour l'Ancien Testament, c'est l'animal impur par excellence et l'attribut privilégié du monde païen et des ennemis d'Israël. Être gardien de pourceaux - ce qui est interdit aux Hébreux est l'image de la déchéance suprême. Le Nouveau Testament conserve cette tradition négative, notamment dans la parabole du fils prodigue qui, après avoir dilapidé tout son bien, est obligé de devenir gardien de cochons (Luc, 15, 11-32).

Car le Nouveau Testament ne réserve pas au porc un sort meilleur que l'Ancien. Les évangiles relatent en détail l'épisode du possédé que le Christ et les apôtres rencontrèrent au pays des Géraséniens. Un grand nombre de démons avaient pris place en lui et l'empêchaient de mener une vie normale car il était constamment hors de son sens, vivant dans des tombes et refusant de porter des vêtements. Jésus ordonna aux démons de sortir de cet homme et d'entrer dans un troupeau de porcs qui paissaient 
dans la montagne toute proche; ce qu'ils firent. Tandis que le possédé retrouvait ses esprits et se mettait à prier, les porcs, au nombre d'environ deux mille, se précipitèrent du haut de la montagne dans le lac de Tibériade (Mat., 8, 30-34; Marc, 5, 9-20; Luc, $8,30-39)$. Ce passage de l'évangile a beaucoup frappé les hommes du Moyen Âge. Il a été repris et commenté par les prédicateurs et les théologiens et a contribué à faire du porc l'un des attributs de Satan. Non seulement le diable prend une forme porcine pour venir tourmenter les hommes et les femmes, mais il grogne comme un goret et, comme lui, il aime à se vautrer dans l'ordure.

Le porc est également pour le christianisme un des attributs des Juifs et de la Synagogue. Par dérision, auteurs et artistes font de l'animal honni des Juifs l'une des figures servant à les désigner. Ce système d'inversion, qui témoigne de la vigueur de l'antijudaïsme médiéval, émerge au tournant des $\mathrm{XII}^{\mathrm{e}}-\mathrm{XIII}{ }^{\mathrm{e}}$ siècles - à un moment où la Chrétienté a tendance à se replier sur elle-même et à se fermer aux cultures voisines - et perdure jusque fort avant dans l'époque moderne. L'iconographie traduit cette attirance supposée des Juifs pour le porc par des formules variées, mais une image prend peu à peu le pas sur toutes les autres : celle qui représente des Juifs, souvent des enfants, en train de téter une truie et d'en absorber les défécations. Née en Allemagne vers le milieu du XIII ${ }^{\mathrm{e}}$ siècle, cette Judensau, d'abord peinte ou sculptée, plus tard gravée et imprimée, se diffusa dans toute l'Europe. Devenue plus rare après le concile de Trente, elle ne disparut cependant pas complètement et resurgit à l'époque contemporaine, notamment dans la propagande nazi.

Attribut de Satan et de la Synagogue, le porc est aussi celui d'un certain nombre de vices personnifiés : d'abord la saleté (sorditas) et la gloutonnerie (gula), plus tard la luxure (luxuria) et la colère (ira); dans ce dernier rôle, toutefois, c'est plutôt le sanglier que le cochon domestique qui est sollicité. Des chapiteaux romans jusqu'aux livres d'emblèmes des $\mathrm{XVI}^{\mathrm{e}}$ et $\mathrm{XVII}^{\mathrm{e}}$ siècles, le porc sert ainsi de monture, de compagnon ou d'attribut à des personnifications allégoriques de chacun de ces vices. Plus simplement encore, il est l'image stéréotypée du péché ou des hommes pécheurs, qui vivent ou se conduisent comme des pourceaux. Dans les sermons des Pères de l'Église comme dans ceux des réformateurs protestants cette comparaison est tellement récurrente qu'elle perd son efficacité. Plus originale est la comparaison qui associe le porc à l'homme relaps ou à celui qui, après avoir confessé ses péchés, retombe dans le vice et dans l'erreur, à l'image de la truie du Nouveau Testament qui «à peine lavée retourne à son bourbier, comme le sot à sa folie et le chien à son vomissement » $(2$ P 2, 22).

\section{Saletés et cochonneries}

Malgré tout le prestige du grand saint Antoine, dont l'attribut principal est un cochon, sa légende et son culte n'ont pas suffi à rendre à tous les porcs un semblant de dignité. En dehors de l'hagiographie, l'animal a conservé sa mauvaise réputation. Cela tient sans doute à sa goinfrerie et à son caractère omnivore. Dès sa naissance, le porcelet se rue vers la mamelle, se bat avec ses congénères pour l'atteindre le premier, essaie d'occuper le maximum de place. Devenu adulte, il passe son temps à chercher de la nourriture, dévore à peu près n'importe quoi, y compris des immondices et des charognes. Par certains côtés, le porc a un instinct carnassier, inconnu chez le bœuf, le mouton ou le cheval. Sa voracité et son aptitude à se nourrir d'ordures en ont fait un animal impur. Il en va 
du reste pareillement de toutes les créatures omnivores : le corbeau, le renard, l'ours et ... l'être humain. Aristote le premier insiste sur le cousinage inquiétant existant entre l'homme et le porc, cousinage confirmé par l'anatomie interne et la physiologie. Plus tard le Christianisme y trouvera matière à paraboles ou à sermons dévalorisant tout ensemble l'homme et le porc. Le cochon a toujours souffert d'être trop semblable à l'homme.

Plus simplement, la symbolique médiévale a fait du porc l'un des attributs ordinaire de la goinfrerie, vice que le latin médiéval exprime par le mot gula, intraduisible en français moderne : le porc est une gueule constamment ouverte, un orifice béant, un gouffre. Il ne regarde jamais vers le ciel - c'est à dire vers Dieu - mais vers le sol, où il espère trouver quelque nourriture. Ce faisant, il participe de la mythologie du monde obscur et souterrain, l'enfer : le porc est un animal infernal. Dans le riche bestiaire de Satan, il occupe une place de choix aux côtés du bouc, de l'ours, du singe, du crapaud, $\mathrm{du}$ serpent et de quelques autres. Encore rare sur les chapiteaux des églises romanes, ce porc infernal devient plus fréquent dans la sculpture gothique et est omniprésent dans la peinture eschatologique de la fin du Moyen Âge. Jérôme Bosch, parmi d'autres artistes, le met fréquemment en scène.

Outre sa voracité il est probable que le porc a également souffert de la couleur sombre de son pelage. Comme le sanglier - la « bête noire » par excellence pour les traités de vénerie - le cochon domestique européen a longtemps présenté une robe non pas rose ou blanche - pour ce faire, il faut attendre le XVIII ${ }^{\mathrm{e}}$ siècle - mais brune, grise, noire, rousse ou tachetée. Aujourd'hui, la plupart des cochons européens sont d'un beau blanc rosé et tout éleveur sait que le porc n'est pas un animal plus sale qu'un autre : il aime l'eau et reste propre s'il dispose d'un espace suffisant et s'il n'a pas trop chaud. Ayant des difficultés à transpirer, il recherche constamment l'eau - ou la boue - pour se rafraîchir. En outre, il n'a pas une acuité visuelle très grande et marche n'importe où, y compris dans la fange. Au Moyen Âge, cette cécité relative a pu passer pour un vice, la vue étant le plus valorisé des cinq sens. Dieu est lumière : tous ceux qui vivent dans les ténèbres, tels les aveugles ou les animaux à mours nocturnes (chouette, chat, renard, crapaud), ne peuvent qu'être que des ennemis de Dieu, des suppôts du Diable. En outre, les deux sens les plus développés chez le porc, l'odorat et le toucher, passent pour les plus grossiers et les plus vils. Le porc est donc une créature brute et stupide, comme le laisse entendre un proverbe ancien, déjà mentionné par l'évangile de Matthieu $(7,6)$ : « Jeter des perles aux pourceaux ».

Attribut de la saleté et de la goinfrerie, le porc n'a en revanche pas été celui de la luxure avant une date relativement récente. D'autres animaux l'ont précédé dans ce rôle : le bouc, l'âne et surtout le chien. Pendant des siècles, de l'Antiquité jusqu'à la fin du Moyen Âge, en matière de lubricité et de sexualité, ce n'est pas le cochon qui fait des « cochonneries » mais le chien, animal tout aussi impur que lui aux yeux des sociétés méditerranéennes, et animal dont le comportement sexuel semble particulièrement sale. La femelle surtout paraît singulièrement lubrique, bien plus que la truie. Pour que le porc devienne sexuellement « cochon », pour qu'il endosse le vice de luxure en plus de tous ses autres vices, il faut vraiment attendre la fin du Moyen Âge, lorsque le chien se valorise et devient définitivement dans les sociétés européennes le fidèle compagnon de l'homme qu'il est resté jusqu'à aujourd'hui. Mais pour que le chien se valorise, il faut le débarrasser de ses impuretés. Concernant la luxure, un transfert symbolique s'opère donc du chien vers le cochon : entre le $\mathrm{Xv}^{\mathrm{e}}$ et le 
$\mathrm{XVII}^{\mathrm{e}}$ siècle ce dernier devient l'animal libidineux par excellence, à la place du chien; désormais tous les hommes qui se livrent à des pratiques obscènes, font des « cochonneries ». En français, le mot prend ce sens vers le milieu du XVII ${ }^{\mathrm{e}}$ siècle; auparavant il désigne simplement le fait d'élever des cochons.

\section{Le porc au tribunal}

Au Moyen Âge, les animaux ont parfois été regardés comme des êtres moraux et perfectibles et par là même responsables de leurs actes. D'où les procès qui leur furent intentés à partir du XIII ${ }^{\mathrm{e}}$ siècle et les peines sévères qui leur furent appliquées comme s'il s'agissait d'êtres humains. En 1266, par exemple, le prévôt de l'abbaye de SainteGeneviève ordonne que soit brûlé vif un porc convaincu d'avoir dévoré un enfant sur le territoire de la paroisse de Fontenay-aux-Roses, dépendante de l'abbaye. Même sentence à Torcy, près de Meaux, en 1274, pour un verrat ayant tué un porcher. En 1408, à Pont-de-l'Arche, en Normandie, un porc qui avait blessé à mort un jeune enfant n'est pas brûlé mais pendu; l'instruction de l'affaire criminelle avait duré vingt-quatre jours, pendant lesquels l'animal fut maintenu en prison. En 1497, au village de Charonne, près de Paris, une truie qui avait blessé un enfant est assommée et dépecée vivante; ses chairs sont jetées aux vents, et son propriétaire et l'épouse de celui-ci sont condamnés à faire un pèlerinage à Notre-Dame de Pontoise « où estant le jour de la Pentecoste ils crieront Merci! et de quoi ils rapporteront certificat ».

Malgré leur immense intérêt, tant sur le plan historique et juridique que sur le plan anthropologique, ces procès d'animaux, que l'on rencontre partout en Europe du XII ${ }^{\mathrm{e}}$ jusqu'au $\mathrm{XVII}^{\mathrm{e}}$ siècle, attendent encore leurs historiens. Ils sont pour l'essentiel restés abandonnés à la " petite histoire » et aux recueils d'anecdotes tournant en dérision les mœurs et les croyances des sociétés anciennes. Attitude anachronique, qui montre que l'on a rien compris à ce qu'était l'Histoire et le nécessaire relativisme culturel qui l'accompagne, mais attitude qui malheureusement, aujourd'hui encore, n'a pas totalement disparu.

$\mathrm{Au}$ Moyen Âge, les porcs ne sont pas les seuls animaux conduits au tribunal. Chiens, chevaux, mulets et bovins sont parfois, eux aussi, soumis à des sentences de justice. En 1314, les juges du comté de Valois condamnent ainsi à la pendaison un taureau qui avait occis un homme d'un coup de corne. Un siècle plus tard, en 1405, à Gisors, un bœuf subit le même sort pour ses seuls " démérites ». Lorsque l'animal estimé coupable n'a pu être identifié ou capturé, il arrive quelquefois que l'on s'empare arbitrairement d'un congénère, qui est emprisonné, jugé et condamné à sa place (en revanche il n'est pas exécuté). Un autre procédé est cependant plus fréquent pour remplacer l'animal coupable qui s'est échappé : il consiste à juger et à supplicier à sa place un mannequin lui ressemblant. Le plus ancien exemple est français et date de 1332. Un cheval avait causé un accident ayant entraîné mort d'homme sur le territoire de la paroisse de Bondy, aux environs de Paris. Cette paroisse relevait de la justice du prieuré de Saint-Martin-des-Champs, réputée fort sévère. Aussi le propriétaire du cheval s'empressa-t-il de conduire l'animal sur un territoire relevant d'une autre juridiction. Mais la ruse fut découverte et l'homme, identifié. Il fut condamné, d'une part à payer une somme équivalente à la valeur d'un cheval, de l'autre à fournir à la justice une « figure de cheval », qui fut traînée et pendue selon le rituel habituel. 
La vedette de ce bestiaire judiciaire n'est cependant pas le cheval mais bien le porc. Dans neuf cas sur dix, c'est lui qui est présent au tribunal. Au point que pour le chercheur, l'histoire de ces procès d'animaux tend à se transformer en une anthropologie historique du cochon. À cette primauté du porc il existe différentes raisons. La principale réside sans doute dans la loi du nombre. Parmi les quadrupèdes domestiques, le porc est en effet l'un des plus abondants en Europe jusqu'à l'époque moderne. Certes, cette population porcine est inégalement répartie et semble diminuer à partir du milieu $\mathrm{du} \mathrm{XVI}^{\mathrm{e}}$ siècle, mais le poids du nombre demeure.

Les porcs sont non seulement très nombreux mais ils sont aussi vagabonds. En ville, nous l'avons vu à propos des cochons de l'ordre des Antonins, ils jouent le rôle d'éboueurs : on en rencontre sur toutes les places, dans toutes les rues, dans tous les jardins et jusque dans les cimetières, où ils cherchent à déterrer les cadavres. Malgré les interdictions des autorités municipales, maintes et maintes fois répétées du XII ${ }^{\mathrm{e}} \mathrm{au}$ $\mathrm{XVIII}^{\mathrm{e}}$ siècle, la divagation des porcs fait partie de la vie quotidienne et occasionne de nombreux accidents.

Toutefois, il est sans doute une autre raison qui explique la présence du porc au tribunal : sa proximité biologique avec l'être humain. Pour les savoirs anciens et médiévaux, l'animal le plus proche de l'homme n'est pas tant l'ours ou le singe que le cochon. Cette proximité biologique est déjà bien connue des médecines grecque et arabe. Une idée récurrente chez beaucoup d'auteurs antiques et médiévaux souligne combien l'organisation anatomique interne de l'homme et celle du cochon sont identiques, ou presque. Ce que confirme en grande partie la médecine contemporaine : anatomiquement et physiologiquement l'homme et le porc sont parents. Au Moyen Âge, dans les écoles de médecine, l'anatomie humaine est ainsi enseignée à partir de la dissection de la truie ou du verrat : l'Église interdisant la mutilation du corps humain après la mort, c'est le cadavre du cochon qui le remplace et remplit des fonctions pédagogiques. À Padoue et à Montpellier, au début du XIV ${ }^{\mathrm{e}}$ siècle, les professeurs et leurs étudiants consomment ainsi annuellement près de cinq cents porcs.

Nous avons conservé plusieurs textes décrivant la dissection d'une truie et s'accompagnant d'un commentaire détaillée des organes internes de la femme, présentés comme identiques à ceux de la truie. Le plus influent provient de la célèbre école de médecine de Salerne, en Italie méridionale. Il a probablement été compilé à la fin $\mathrm{du} \mathrm{XI}^{\mathrm{e}}$ siècle et s'appuie sur un jeu de mots latins exprimant parfaitement ce cousinage $:$ porcus $=$ corpus. . Cette anagramme se retrouve dans plusieurs textes médicaux jusqu'au XVII ${ }^{\mathrm{e}}$ siècle. Il faut cependant signaler que dès la fin du Moyen Âge, d'abord en Italie puis dans le reste de l'Europe, les interdits de l'Église se firent moins drastiques et que les médecins purent commencer à disséquer le corps humain - notamment celui de condamnés fraîchement exécutés - et non plus celui du cochon pour enseigner l'anatomie à leurs étudiants. 